\title{
A pilot study of positive mood induction in euthymic bipolar subjects compared with healthy controls
}

\author{
ANNE FARMER*, DOMINIC LAM, BARBARA SAHAKIAN, JONATHON ROISER, \\ AILBHE BURKE, NATHAN O’NEILL, SAM KEATING, GEORGIA POWELL SMITH \\ AND PETER MCGUFFIN \\ MRC Social, Genetic and Developmental Psychiatry Research Centre, Institute of Psychiatry, London, UK
}

\begin{abstract}
Background. Demonstrating differences between euthymic bipolar subjects and healthy controls in response to positive (happy) mood induction may help elucidate how mania evolves. This pilot study evaluates the Go task in a reward paradigm as a method for inducing a happy mood state and compares the response of euthymic bipolar subjects and healthy controls.
\end{abstract}

Method. The Sense of Hyperpositive Self Scale, the Tellegen positive and negative adjectives, the Global-Local task and a visual analogue scale for measuring positive affect were administered to 15 euthymic bipolar subjects and 19 age-and-sex-matched healthy control subjects before and after they had performed the Go task in a reward paradigm.

Results. Significant differences were found between subjects and controls on several measures at each time-point but there were no differences across the groups across time except for the visual analogue scales, where subjects had a more sustained duration in self-reported happiness compared with controls.

Conclusions. This pilot study has shown that a positive affect can be induced in bipolar subjects and controls which can be demonstrated by changes in scores on several tasks. However, only the visual analogue scales showed a significant difference between cases and controls over time. Such tests may prove valuable in furthering understanding about the evolution of manic mood states.

\section{INTRODUCTION}

Much research has focused on cognitive aspects of depression but manic mood states, although they have been far less intensively investigated, also appear to be associated with altered cognitions (Chamberlain \& Sahakian, 2004). Recent studies have shown that changes in the fluency of thought, speech, learning and memory impairment as well as disturbances in association patterns and attentional processes occur in the acute phase of mania, and persist in remission in between one-quarter and

\footnotetext{
* Address for correspondence: Professor Anne Farmer, Box 080 , MRC Social, Genetic and Developmental Psychiatry Research Centre, Institute of Psychiatry, De Crespigny Park, Camberwell, London SE5 8AF, UK.

(Email: a.farmer@iop.kcl.ac.uk)
}

one-third of subjects (Martinez-Aran et al. 2004; Thompson et al. 2005). However, standard emotionally neutral tests of motor function, memory and executive function do not differentiate between the cognitive changes associated with mania from those associated with depression. Consequently Sahakian and colleagues (Roiser et al. 2003; Chamberlain \& Sahakian, 2004) have suggested that 'hot' or emotion-dependent tasks may provide the key to the cognitive changes specific to each mood state.

While there have been several studies examining the cognitive changes associated with negative mood induction in both healthy students and subjects with depression, examination of the cognitions associated with mania 
in response to positive mood induction has been a relatively neglected area of research. One exception is a study by Wright and colleagues (Wright et al. 2005). The authors found that, after positive mood induction using video clips, bipolar patients scored significantly higher on a 24-item dysfunctional attitudes scale than remitted depressives and a normal control group. However, one of the problems with a video clips paradigm is that it can lead to cognitive priming. Hence a more cognitively neutral paradigm may be advantageous. The following pilot study was undertaken to examine whether it was possible to induce a positive mood using the Go task in a reward paradigm and whether once induced, significant differences in the cognitions of euthymic bipolar subjects compared with healthy controls could be demonstrated.

\section{METHOD}

Fifteen subjects who had participated in a genetic case-control study of bipolar disorder and who fulfilled operational criteria for DSM-IV bipolar 1 disorder volunteered to participate in a pilot mood induction study. All bipolar subjects had been previously interviewed using the Schedules for the Clinical Assessment of Neuropsychiatry (SCAN; Wing et al. 1990) for psychopathology occurring in their worst episode of mania and their worst episode of depression and the results entered into the CATEGO5 scoring program (Celik, 2003) to assign a lifetime-ever diagnosis.

The Past History Schedule (McGuffin et al. 1986) was administered by phone to establish whether 19 volunteer age- and sex-matched control subjects had ever suffered or were currently suffering from any mental disorder.

All participants gave written informed consent and were screened with the Beck Depression Inventory (BDI; Beck et al. 1961) and the Altman Mania Scale (AMS; Altman et al. 1997) by telephone one week prior to testing, as well as on the day of the mood induction procedure. (The short-form BDI was administered by phone and the long-form completed on the day of testing.)

On the day of testing, all participants also completed the National Attainment Reading Test (NART; Nelson, 1982) to provide a proxy measure of IQ. In addition, they also undertook the following before and after completing the Go task (Johnson et al. 2005) in a reward paradigm:

(1) The Sense of Hyper-Positive Self Scale (SHPSS; Lam et al. 2005b). Subjects are asked to describe, first how they are 'most of the time' and second how they 'would like to be' by responding to seven positive adjectives: confident, dynamic, etc., on a sixpoint scale from 'not at all' to 'extremely'.

(2) Tellegen's Mood Adjective checklist (Watson et al. 1988). Subjects rate 10 adjectives of high positive affect and 10 of high negative affect a five-point scale rating from 'not true at all' to 'very true about me'.

(3) The Global-Local processing task (Witkin et al. 1971; Shah \& Frith, 1983). The ability to identify whether a small figure is embedded in a larger one.

(4) Visual analogue scales on four occasions throughout the testing period (subjects placed a cross on a $10 \mathrm{~cm}$ line to indicate how happy or sad they felt.

The Go task was programmed using a serial response box with five lamps and five corresponding buttons. Subjects started each trial by pressing a centre button, after which a randomly selected lamp lit. The subject is then asked to press the button below that lamp as fast as possible. Subjects' reaction times were recorded in a computerized program. A practice trial, consisting of five button pressings was followed by two 2-minute button pressings without any monetary rewards (neutral condition). Subjects were then given feedback that their speed was 'very fast'. After these trials in neutral condition, subjects were encouraged to press as many buttons as they could in 2 minutes, and were paid 10 pence for each button pressed correctly. The program was set so that subjects were given feedback that they performed accurately $70 \%$ of the time.

\section{RESULTS}

Age, gender, BDI, Altman Mania Scale (AMS), NART and overall Go task scores for cases and controls

The mean age for 15 cases was 44.4 years [standard deviation (S.D.) $=13.42$ years] and for 19 controls was 35.42 years (s.D. $=13.60$ years). 
Table 1. Mean scores, standard deviations and significance levels for cases and controls for measures taken pre- and post-mood induction

\begin{tabular}{|c|c|c|c|c|c|c|}
\hline \multirow[b]{2}{*}{ Test } & \multicolumn{2}{|c|}{ Pre-mood induction } & \multirow{2}{*}{$\begin{array}{c}\text { Between- } \\
\text { subject } \\
\text { significance level }\end{array}$} & \multicolumn{2}{|c|}{ Post-mood induction } & \multirow{2}{*}{$\begin{array}{c}\text { Between- } \\
\text { subject } \\
\text { significance level }\end{array}$} \\
\hline & Cases & Controls & & Cases & Controls & \\
\hline SHPSS overall score (S.D.) & $58 \cdot 21(10 \cdot 50)$ & $61 \cdot 53(7 \cdot 47)$ & N.S. & $57 \cdot 00(9 \cdot 28)$ & $59 \cdot 89(7 \cdot 16)$ & N.S. \\
\hline Tellegen positive adjectives (s.D.) & $31 \cdot 14(7 \cdot 33)$ & $28 \cdot 79(7 \cdot 31)$ & N.S. & $32 \cdot 07(6 \cdot 24)$ & $28 \cdot 47(6 \cdot 70)$ & N.S. \\
\hline Tellegen negative adjectives (s.D.) & $13 \cdot 29(2 \cdot 76)$ & $11 \cdot 16(1 \cdot 74)$ & $p=0 \cdot 02$ & $11 \cdot 87(2 \cdot 00)$ & $10 \cdot 63(1 \cdot 17)$ & $p=0 \cdot 05$ \\
\hline Global-Local time taken (s.D.) & $208 \cdot 99(111 \cdot 40)$ & $123 \cdot 01(61 \cdot 49)$ & $p=0 \cdot 01$ & $157 \cdot 08(92 \cdot 53)$ & $84 \cdot 27(47 \cdot 25)$ & $p=0 \cdot 01$ \\
\hline
\end{tabular}

Sixty-seven per cent of cases and $84 \%$ of controls were female. The mean score on the short-form BDI undertaken 1 week prior to testing was 2.60 (S.D. $=2.92$ ) for cases and 1.00 (S.D. $=1.77)$ for controls. Altman Mania Scale (AMS) scores were 1.87 (S.D. $=1.77$ ) for cases and 1.93 (s.D. $=1.75$ ) for controls. NART scores were 39.80 (S.D. $=5.88$ ) for cases and 39.95 (S.D. $=5 \cdot 78)$ for controls. There were no significant differences between cases and controls for age, sex, BDI (short form), AMS or NART.

However, there were significant differences between cases and controls for the long version of the BDI undertaken on the day of testing. Mean BDI for cases was 5.00 (s.D. $=5.98$ ) and for controls was 1.37 (s.D. $=2 \cdot 67$ ) $[t$ test $(t)=2 \cdot 23$, degrees of freedom (df) $17 \cdot 19, p=$ $0 \cdot 04]$. Repeated measure analysis of variance (ANOVA) showed significant differences between cases and controls for BDI score over time $(F=8.87, p<0 \cdot 01)$ but there was no significant group by time interaction $(F=3 \cdot 19, p=$ N.S. $)$.

For the Go task, cases made significantly fewer responses compared with controls. Mean number of responses for cases was 399.29 (S.D. $=61 \cdot 48)$ and for controls was $460 \cdot 00$ $($ S.D. $=42 \cdot 98) \quad(t=-3 \cdot 12, \quad \mathrm{df}=22 \cdot 59, \quad p<0 \cdot 01)$. However when age was added to the analysis as a covariate, the significant group difference disappeared (group: $F=2 \cdot 11, p=$ N.S.; age: $F=17.53, p<0 \cdot 01)$.

Cases were also significantly slower than controls for both the non-reward as well as the rewarded 2 minutes. Average response time, in milliseconds, at the non-rewarded 2 minutes for cases was $630 \cdot 60$ (S.D. $=113 \cdot 00)$ and for controls was $542.13($ s.D. $=61 \cdot 74)(t=2 \cdot 62, \mathrm{df}=19 \cdot 23$, $p=0.02$ ). For the rewarded 2 minutes average response time, in milliseconds, for cases was $627 \cdot 30$ (S.D. $=111 \cdot 36$ ) and for controls was
$552 \cdot 07 \quad($ S.D. $=59 \cdot 97)(t=2 \cdot 27, \quad \mathrm{df}=19 \cdot 07, \quad p=$ $0 \cdot 04)$. However, these significant group differences again disappeared when age was added as a co-variate (non-reward 2 minutes: group: $F=1 \cdot 15, p=$ N.S.; age: $F=13.42, p<0.05$; reward 2 minutes: group: $F=0 \cdot 36, p=$ N.S.; age: $F=15 \cdot 86, p<0 \cdot 01)$. There were no significant differences between cases and controls for percentage correct, percentage wrong or percentage timed out.

\section{SHPSS, Tellegen adjectives and Global-Local task in cases and controls pre- and post-mood induction}

The mean scores on these measures pre- and post-mood induction (MI) are shown in Table 1.

For the SHPSS, Table 1 shows that there was no significant difference in overall scores between cases and controls for either pre- or postMI. Also repeated measures ANOVA with age as a co-variate indicates that there was no effect of MI on scores for both cases or controls $(F=1 \cdot 13, \quad p=$ N.s. $)$ and no MI by group $(F=0.18 ; p=$ N.S. $)$ or MI by age interaction $(F=0 \cdot 13, p=$ N.S. $)$.

Table 1 shows that cases endorsed significantly more Tellegen negative adjectives as being true about themselves than controls both pre- $(t=2.54, \mathrm{df}=20.48, p=0.02)$ and post$(t=2 \cdot 13, \mathrm{df}=21 \cdot 35, p=0 \cdot 05)$ mood induction, although there were no significant differences between cases and controls for positive adjectives. For negative adjectives there is an MI effect of lowering scores $(F=9 \cdot 81, p<0.01)$ in both cases and controls but there is no significant MI by group $(F=1 \cdot 26, p=$ N.s. $)$ or MI by age interaction $(F=0 \cdot 16 ; p=$ N.s. $)$.

On the Global-Local task, Table 1 shows that there were significant differences between cases and controls on both testing occasions for both 
Table 2. Mean scores on four visual analogue (VA) scales for cases and controls

\begin{tabular}{cllll}
\hline \hline & VA1 $^{\mathrm{a}}$ & VA2 & VA3 & VA4 \\
\hline Cases & $50 \cdot 75$ & $58 \cdot 67$ & $58 \cdot 53$ & $60 \cdot 00$ \\
(s.D.) & $(12 \cdot 84)$ & $(15 \cdot 75)$ & $(14 \cdot 62)$ & $(18 \cdot 77)$ \\
Controls & $57 \cdot 68$ & $63 \cdot 56$ & $64 \cdot 16$ & $56 \cdot 74$ \\
(s.D.) & $(13 \cdot 60)$ & $(15 \cdot 57)$ & $(14 \cdot 82)$ & $(13 \cdot 00)$ \\
\hline \hline
\end{tabular}

${ }^{a}$ Go task in reward condition occurred following this rating.

average time taken (pre-MI: $t=2 \cdot 67, \mathrm{df}=20 \cdot 90$, $p=0 \cdot 01$; post-MI: $t=2 \cdot 78, \mathrm{df}=19 \cdot 71, p=0 \cdot 01)$ and average number correct (pre-MI: $t=-2 \cdot 11$, $\mathrm{df}=15 \cdot 64, \quad p=0 \cdot 05 ; \quad$ post-MI $: \quad t=-2 \cdot 63$, $\mathrm{df}=14 \cdot 71, p=0 \cdot 02)$. Both cases and controls improved their speed at the task following MI $(F=4.36, p=0.05)$ and there was also a significant effect of age on speed over time $(F=15 \cdot 00$, $p<0.01)$ but there were no significant group by MI $(F=0.00, p=$ N.S. $)$ or age by MI $(F=3 \cdot 48$, $p=$ N.s.) interactions. Both cases and controls improved on the number correct but this was not significant $(F=3 \cdot 72, p=0 \cdot 06)$, although the effect due to age was $(F=6.52, p=0.02)$. There were no interactional effects for group by MI $(F=0.35, p=$ N.S. $)$ or for MI by age $(F=1.73$, $p=$ N.S.).

\section{Visual analogue scales pre- and post-mood induction}

The mean scores on the visual analogue (VA) scales 1 through 4 for cases and controls are shown in Table 2. The Go task in the reward paradigm came between VA ratings 1 and 2 . The table shows that cases rated themselves as somewhat less happy than controls before MI although this was not statistically significant. Mean scores for both cases and controls increased following MI, indicating that all subjects were happier. However, again there were no statistically significantly score differences between cases and controls. After completion of the second Global-Local task (VA4), the mean VA scores for controls had returned to just below that of the start of testing while mean scores for cases remained elevated. This was reflected in the repeated measures ANOVA that showed that both cases and controls became happier following $\mathrm{MI}(F=4 \cdot 01, p=0 \cdot 05)$ and that there was a significant $\mathrm{MI}$ by group interaction $(F=4 \cdot 51, p=0 \cdot 04)$.

\section{DISCUSSION}

The SHPSS showed no differences in the scores of cases and controls at either time of testing although repeated measures ANOVA has shown that scores for all subjects became more positive following MI. However cases and controls did not differ in the magnitude of positive self-regard following MI.

In contrast, the Tellegen negative adjectives, and the Global-Local task show significant differences between cases and controls, at both times of testing. Such case-control differences have been shown in other studies of euthymic bipolar subjects (Thompson et al. 2005). In addition, in the Global-Local task, repeated measures ANOVA showed that all subjects had an enhanced ability to perform this task both correctly and speedily following MI, which could have been due to a practice effect rather than the impact of mood elevation. However, although both the Tellegen and Global-Local tasks showed greater improvement in scores in cases compared with controls following MI (the effect predicted by our hypothesis), these failed to achieve statistical significance, which may have been due to the small sample size of the pilot study.

The VA scales provide a simple self-rating of 'happiness' which has shown a difference in response to positive MI between cases and controls. Table 2 shows that although cases were unhappier at the start of the testing procedure compared with controls, their scores rose following MI and remained elevated while controls show a rise in happiness ratings which declined again back to (below) preinduction levels by the end of the testing procedure. This finding of a prolonged happy mood response after positive $\mathrm{MI}$ is consistent with Goplerud \& Depue's (1985) finding that cyclothymic participants had a more prolonged duration of recovery of mood, behaviour and cognitions than normal controls after a naturalistic stressful event. Although we have not shown any statistically significant differences between cases and controls for individual visual analogue ratings, repeated measures ANOVA showed that the change over time in ratings was significant.

The MI paradigm employed in this study has demonstrated not only that both cases and 
controls respond to positive MI but also that the response shown by euthymic bipolar subjects is different to that of controls in being more sustained. Thus our findings support Depue's Behavioural Activation theory (BAS) theory of bipolar disorder (Depue et al. 1981). An alternative explanation is that the euthymic bipolar subjects started from a lower baseline and consequently had more scope for mood elevation. Indeed, VA1 shows that bipolar subjects do have lower scores than the controls although these do not differ significantly. However, what is particularly notable about the VA scores over time is that mood elevation persists in bipolar subjects so that by VA4 bipolar subjects scores are remaining high while scores for controls have come down to pre-MI levels. Thus we suggest that it is the sustained elevated mood following positive MI that distinguishes bipolar subjects from controls rather than the level of their self-reported mood prior to MI.

However, our results need to be considered in light of some limitations to the study design. First, we have not included a psychiatric control group, for example subjects with remitted unipolar depression, which would have allowed the identification of vulnerability factors that are unique to bipolar disorder. Secondly, in order to distinguish between the effects of mood change and a practice effect, a negative MI paradigm could have been added to the positive MI, counterbalancing the order of presentation. Thirdly, we have not included a control condition for unrewarded activity. Hence we cannot say for certain that the mood improvement was entirely due to the rewarding properties of the Go task.

Despite these caveats and the small size of a pilot study, we suggest that our results show that following positive MI, significant differences can be found between the response of euthymic bipolar subjects and healthy controls. A detailed analysis of the changes in mood, behaviour and cognition that occur in an elevated mood state will improve our understanding of how mania unfolds. Not only will this have important implications for the development of psychological treatments for bipolar disorder (Lam et al. 2005a) but also the MI paradigm used in this pilot study is relatively simple and could be readily employed for examining the brain function in mania using neuroimaging techniques.

\section{CONCLUSIONS}

The results of this study have shown that a positive or 'happy' mood can be induced in both control and euthymic bipolar subjects using the Go Task in a reward paradigm which can be demonstrated by increases in 'happiness' ratings on scales such as the SHPSS, Tellegen adjectives, the Global-Local task and serial visual analogue ratings. However, only the visual analogue ratings showed significant differences between cases and controls following MI, with cases having a sustained elevation of mood while in controls mood returns to normal.

\section{DECLARATION OF INTEREST}

The subjects who agreed to participate in this pilot study had already participated in a casecontrol genetic study funded by Glaxo Smith Kline Research and Development. Anne Farmer and Peter McGuffin have received consultancy fees and honoraria for presentations, chairing, and participating in expert panels, plus travel and subsistence costs from Glaxo Smith Kline, Eli Lilly, Bristol Myers Squibb and Lundbeck.

\section{REFERENCES}

Altman, E. G., Hedeker, D., Peterson, J. L. \& Davis, J. M. (1997). The Altman Self-Rating Mania Scale. Biological Psychiatry 42, 948-955.

Beck, A. T., Ward, C. H., Mendelson, M., Mock, J. \& Erbaugh, J. (1961). An inventory for measuring depression. Archives of General Psychiatry 4, 561-571.

Celik, C. (2003). Computer assisted personal interviewing application for the Schedules for Clinical Assessment in Neuropsychiatry, Version 2.1, and diagnostic algorithms for WHO ICD-10 Chapter V $D C R$ and for statistical manual $I V$. Release 1. Ed. 1.0.3.5. Win 9x NT. World Health Organisation: Geneva.

Chamberlain, S. R. \& Sahakian, B. J. (2004). Cognition in mania and depression: psychological models and implications. Current Psychiatry Reports 6, 451-458.

Depue, R. A., Slater, J. F., Wolfstetter-Kausch, H., Klein, D., Goplerud, E. \& Farr, D. (1981). A behavioral paradigm for identifying persons at risk for bipolar depressive disorder: a conceptual framework and five validation studies. Journal of Abnormal Psychology 90, 381-437.

Goplerud, E. \& Depue, R. A. (1985). Behavioural response to naturally occurring stress in cyclothymia and dysthymia. Journal of Abnormal Psychology 94, 128-139.

Johnson, S. L., Ruggero, C. J. \& Carver, C. S. (2005). Cognitive, behavioral, and affective responses to reward: links with 
hypomanic symptoms. Journal of Social and Clinical Psychology 24, 894-906.

Lam, D. H., Hayward, P., Watkins, E. R., Wright, K. \& Sham, P. $(2005 a)$. Relapse prevention in patients with bipolar disorder: cognitive therapy outcome after 2 years. American Journal of Psychiatry 162, 324-329.

Lam, D. H., Wright, K. \& Sham, P. (2005b). Sense of hyper-positive self and response to cognitive therapy in bipolar disorder. Psychological Medicine 35, 69-77.

Martinez-Aran, A., Vieta, A., Colom, F., Torrent, C., SanchezMoreno, J., Reinares, M., Benabarre, A., Goikolea, J. M., Brugue, E., Daban, C. \& Salamero, M. (2004). Cognitive function across manic or hypomanic depressed and euthymic states in bipolar disorder. American Journal of Psychiatry 161, 262-270.

McGuffin, P., Katz, R. \& Aldrich, J. (1986). Past and present state examination: the assessment of 'lifetime ever' psychopathology. Psychological Medicine 16, 461-465.

Nelson, H. E. (1982). National Adult Reading Test, NART. Nelson: Windsor.

Roiser, J. P., Rubensztein, J. S. \& Sahakian, B. J. (2003). Cognition in depression. Psychiatry 2, 43-47.
Shah, A. \& Frith, U. (1983). An islet of ability in autistic children: a research note. Journal of Child Psychology and Psychiatry 24, 613-620.

Thompson, J. M., Gallagher, P., Hughes, J. H., Watson, S., Gray, J. M., Ferrier, I. N. \& Young, A. H. (2005). Neurocognitive impairment in euthymic patients with bipolar affective disorder. British Journal of Psychiatry 186, 32-40.

Watson, D., Clark, L. A. \& Tellegen, A. (1988). Development and validation of brief measures of positive and negative affect: the PANAS Scale. Journal of Personality and Social Psychology 54, 1063-1070.

Wing, J. K., Babor, T., Brugha, T., Burke, J., Cooper, J. E., Giel, R., Jablenski, A., Regier, D. \& Sartorius, N. (1990). SCAN. Schedules for Clinical Assessment in Neuropsychiatry. Archives in General Psychiatry 47, 589-593.

Witkin, H. A., Ottman, P. K., Raskin, E. \& Karp, S. (1971). A Manual for the Embedded Figure Test. Consulting Psychologists Press: Mountain View, CA.

Wright, K., Lam, D. H. \& Newsom-Davis, I. (2005). Induced mood and dysfunctional attitudes in remitted bipolar I affective disorder. Journal of Abnormal Psychology 114, 689-696. 